\title{
Erratum to: Is 1,25-Dihydroxyvitamin D3 Receptor Expression a Potential Achilles' Heel of CD44+ Oral Squamous Cell Carcinoma Cells?
}

\author{
Martin Grimm ${ }^{1} \cdot$ Beshr Shokri $^{1}$ - Dorothea Alexander ${ }^{1}$ - Adelheid Munz ${ }^{1}$. \\ Juergen Hoffmann ${ }^{2} \cdot$ Siegmar Reinert ${ }^{1}$
}

Published online: 26 July 2017

(C) Springer International Publishing AG 2017

Erratum to: Targ Oncol (2013) 8:189-201

DOI 10.1007/s11523-013-0255-Z

In the published version of the article, Beshr Shokri was not listed as an author but included in the Acknowledgement statement.

The updated author list is as follows:

Martin Grimm, Beshr Shokri, Dorothea Alexander, Adelheid Munz, Juergen Hoffmann, Siegmar Reinert

The updated Acknowledgements and Conflict of interests statements should read:

Acknowledgements We thank Julia Fertinger and Mohammed Saleh for their technical assistance.

\section{Compliance with Ethical Standards}

Conflict of Interest The authors declare that they have no competing interests.

The online version of the original article can be found at http://dx.doi.org/ 10.1007/s11523-013-0255-Z

Martin Grimm

dr.dr.martingrimm@googlemail.com

1 Department of Oral and Maxillofacial Surgery, University Hospital Tuebingen, Osianderstrasse 2-8, 72076 Tuebingen, Germany

2 Department of Oral and Maxillofacial Surgery, University Hospital Heidelberg, Im Neuenheimer Feld 400, 69120 Heidelberg, Germany 